\title{
Perioperative nursing principles guided by the concept of enhanced recovery after surgery ${ }^{\dagger}$
}

\author{
Hong-Jie Xie ${ }^{a}$, Fan Cui ${ }^{a}$, Wei-Bing Shuang ${ }^{\mathrm{b}, *}$ \\ ${ }^{a}$ Department of First Clinical Medical College, Shanxi Medical University, Taiyuan, Shanxi 030001, China \\ ${ }^{b}$ Department of Urology, The First Hospital of Shanxi Medical University, Taiyuan, Shanxi 030001, China
}

Received: 18 July 2020; Accepted: 31 August 2020; Published: 20 March 2021

\begin{abstract}
Objective: To explore the clinical effect of perioperative nursing guided by the concept of enhanced recovery after surgery and summarize them.

Methods: Pubmed, Chinese National Knowledge Infrastructure (CNKI), Chinese Biomedical Literature Database (CBM), Wanfang Database, and VIP Database were searched to obtain the relevant literature involving enhanced recovery after surgery (ERAS) guidance, obtain the effective clinical data, review the reports in literature, and obtain the effective scheme.

Results: Compared with the traditional nursing program, perioperative nursing principles guided by the concept of ERAS provide more accurate nursing care to patients and reduce the occurrence of intraoperative stress events through comprehensive nursing measures such as preoperative pre-rehabilitation measures, intraoperative body temperature and fluid management, postoperative analgesia, prevention of nausea and vomiting, early mobilization, catheter nursing, and better out-of-hospital follow-up.

Conclusions: Perioperative nursing principles guided by the concept of ERAS can significantly reduce the incidence of perioperative complications, shorten the hospital stay of patients, and promote postoperative rehabilitation of patients. The transformation and implementation of this concept can bring significant benefits to hospitals, medical care, and patients.
\end{abstract}

Keywords: enhanced recovery after surgery $\bullet$ perioperative care $\bullet$ prehabilitation measures $\bullet$ intraoperative optimization measures • postoperative rehabilitation measures

(c) Shanxi Medical Periodical Press.

\section{Introduction}

Because the traditional care model currently cannot meet the patient's disease care needs, healthcare workers have proposed the concept of enhanced recovery after surgery (ERAS). The care model guided by this new concept is achieved by optimizing measures to reduce the psychological/physical traumatic stress of surgical patients, thereby reducing the incidence of postoperative complications and

+ This project was supported by Emerging industry leading talent project of Shanxi Province (No. [2020]587).

How to cite this article: Xie HJ, Cui F, Shuang WB. Perioperative nursing principles guided by the concept of enhanced recovery after surgery. Front Nurs. 2021;1:1-6. 
mortality. ${ }^{1-3}$ Studies have shown that compared with the traditional clinical management, the clinical nursing model guided by ERAS can significantly reduce postoperative complications, shorten hospital stay, and reduce the readmission rate of patients. ${ }^{4,5}$ With the development of evidence-based medicine, the concept of ERAS has been widely used in surgical departments. Through the improvement of traditional clinical nursing programs, it provides a good nursing guarantee for patients during perioperative period and promotes the postoperative recovery of patients. In this article, effective clinical data were obtained by searching related literature, and effective schemes were summarized. The detailed descriptions are given in Section 2.

\section{Prehabilitation measures}

Prehabilitation measures have developed from preoperative preparation measures, which mainly refer to psychological counseling and health education, nutritional support, and related lifestyle training from the process of disease diagnosis to treatment to improve the patient's surgical tolerance and reduce the occurrence of intraoperative and postoperative complications. ${ }^{6}$

\subsection{Mental health interventions}

Due to the concern of surgical patients about the uncertainty of surgical treatment prognosis and surgical risk, the body will involuntarily increase the perioperative stress response of patients through neuro-endocrine, inflammatory, and other means, thus increasing the risk of surgery and the incidence of postoperative complications. ${ }^{7,8}$ ERAS idea guiding principles of perioperative nursing by thought that by doctors, nurses and anesthesia and other multidisciplinary personnel on health education to patients and perioperative psychological counseling is necessary, for planning operations in patients with psychological counseling can alleviate the anxiety and depression, reduce stress reaction, and accelerate the postoperative recovery of patients. ${ }^{9}$ Preoperative education mainly includes the communication of specific conditions, guidance of lifestyle, explanation of anesthesia methods, prevention and control of related complications, postoperative analgesic strategies, related strategies at each stage of rehabilitation, and other aspects. ${ }^{10}$ Written text, multimedia courseware, or three-dimensional models can be used during education, which not only facilitates patients to understand surgery-related problems but also helps to reduce patients' anxiety about surgery and has a positive impact on prognosis. ${ }^{11}$

\subsection{Evaluation and optimization of preoperative basic conditions}

\subsubsection{Preoperative smoking and drinking are prohibited}

Poor lifestyle habits will increase poor outcomes in surgical patients. Pierre et al. ${ }^{12}$ stated that patients who smoked before surgery had significantly increased odds of in-hospital mortality and complications. Smoking cessation more than 4 weeks before surgery can significantly reduce intraoperative and postoperative respiratory complications and effectively improve the healing of surgical incisions. Preoperative alcohol consumption can also affect the prognosis of surgery. Alcohol consumption not only affects the prophylactic use of antibiotics but also threatens life because of the use of cephalosporins. Stopping drinking has a positive promoting effect on reducing postoperative complications and reducing the risk of postoperative infection. ${ }^{13,14}$

\subsubsection{Preoperative management of underlying diseases and medications}

Poor preoperative control of underlying diseases and improper use of drugs will not only reduce immune function and anti-infective ability but also interfere with tissue repair and affect wound healing. Therefore, young patients without other complications and elective surgery are mostly selected in the early stage of fast track surgery (FTS). The effective management and control of underlying diseases can relieve inflammatory reactions, improve the immune function of the body, protect the intestinal mucosal barrier function, and then reduce the occurrence of complications such as postoperative infection. Therefore, patients with advanced age, malnutrition, and multiple underlying diseases are also gradually included in the management of ERAS scope. ${ }^{15,16}$ In the process of preoperative optimal management of some specific surgical diseases, not only the idea of management and control must be implemented but also the concept of regular supplements must be practiced. For example, in the preoperative preparation of adrenal pheochromocytoma, it is necessary to effectively control hypertension and tachycardia before operation, actively expand blood volume, and make sufficient preparation for safely passing the operation period.

\subsection{Preoperative nutritional intervention}

Studies have confirmed that severe preoperative malnutrition is an independent risk factor leading to surgical complications, mortality, and slowing of postoperative 
rehabilitation of patients. Preoperative nutritional support can improve the patient's surgical tolerance, reduce the occurrence of complications such as postoperative infection, and accelerate the patient's postoperative rehabilitation. ${ }^{17}$ The main contents of nutritional support are to reduce disease-related symptoms, control blood glucose, and optimize physical status. ${ }^{18}$ Some nutritional screening tools such as the Nutritional Risk Score (NRS2002) and the Subjective Assessment of Global Nutritional Status (PG-SGA) have been applied, or simply according to BMI > 18.5, serum albumin $>30 \mathrm{~g} / \mathrm{L}$, and hemoglobin $>80 \mathrm{~g} / \mathrm{L}$ as the bottom line of nutritional status. The daily protein intake should be $1.2 \mathrm{~g} / \mathrm{kg}$, and the protein deficient in the conventional diet is supplemented with whey protein.

\subsection{Preoperative diet and bowel preparation}

Traditional preoperative dietary guidance suggests that patients should fast $12 \mathrm{~h}$ before surgery and stop drinking for 4-6 h to fully empty the stomach to effectively prevent the chance of respiratory obstruction and lung injury from intraoperative aspiration. ${ }^{19,20}$ However, prolonged preoperative dietary deprivation can lead to metabolic stress in patients, poor tolerance to surgery, and postoperative adverse effects such as dehydration, hypoglycemia, and insulin resistance..$^{19,21,22}$ At present, for urological surgery, it is recommended to fast for $6 \mathrm{~h}$ before surgery and stop drinking for $2 \mathrm{~h}$ to improve the patient's tolerance to surgery, reduce insulin resistance, and perioperative stress response.

\section{Intraoperative nursing measures}

\subsection{Intraoperative temperature management}

The patient's body temperature was maintained $>36^{\circ}$ to prevent complications of hypothermia. Studies have shown that anesthetic drugs, long operation time, massive blood loss, infusion and continuous fluid irrigation during related surgeries can cause intraoperative hypothermia. Hypothermia can lead to coagulation and immune dysfunction, increase the occurrence of intraoperative bleeding, cardiovascular and cerebrovascular accidents, induce postoperative infection, delay wound healing, and delay drug metabolism leading to prolonged postoperative recovery time. ${ }^{23,24}$ ERAS reduces hypothermic injury and accelerates patient recovery by using active insulation measures such as increasing preoperative core temperature, ensuring appropriate operating room temperature, warming of lavage fluid, and active postoperative insulation.

\subsection{Intraoperative fluid management}

Intraoperative reasonable fluid monitoring ensures circulating blood volume and maintains blood perfusion of the heart, intestine, and kidneys, but excessive fluid infusion often causes intestinal tissue edema, cardiopulmonary and coagulation dysfunction, and prolongs intestinal recovery time. At present, the clinical fluid replacement methods mostly use open, restrictive and goal-directed fluid therapy (GDFT), but each has its own shortcomings. Open fluid replacement leads to weight gain and postoperative complications. Restrictive fluid replacement can effectively shorten the recovery time of gastrointestinal function, reduce cardiopulmonary complications, and improve wound healing. ${ }^{25}$ But it will lead to insufficient circulating blood volume, affecting the intestinal tract. On this basis, the ERAS concept recommends GDFT, which can optimize organ perfusion and oxygen volume supply effectively reduce the incidence of infection, intestinal paralysis, intestinal obstruction, nausea and vomiting and improve $\mathrm{PH}$ value through monitoring indicators such as stroke volume variability (SVV) and arterial pulse pressure variability (PPV). ${ }^{26}$ When selecting the type of fluid supplementation, crystalloids can effectively supplement physiological requirements, but the effect of maintaining blood volume is poor, and interstitial edema and pulmonary edema are more likely to occur; colloid fluid expansion effect is better, which can reduce tissue edema, but it may interfere with the coagulation effect and cause allergic reactions. Therefore, colloid volume expansion is preferred when the patient develops hypotension, and crystalloids are preferred if only physiological requirements need to be supplemented. In addition, hypotension due to vasodilatation caused by anesthetic drugs should be discerned, and vasopressors are preferred in such cases. ${ }^{27}$

\section{Postoperative rehabilitation measures}

\subsection{Effective postoperative analgesia}

Pain not only causes adverse emotions and sleep disorders but also causes a series of adverse reactions such as nausea, intestinal paralysis, and postoperative infection. It affects the early postoperative ambulation of patients and the early postoperative recovery of patients. ${ }^{28}$ Opioids are mostly used for previous analgesia, which leads to excessive sedation, risk of addiction, inhibition of recovery of intestinal function, and increased occurrence of postoperative 
complications. Opioids are also less than ideal in terms of analgesic effect. The individualized analgesic regimen of preventive analgesia, precise analgesia, and multimodal analgesia has been gradually developed in clinical practice to ensure that the adverse reactions of related drugs are reduced while rationalizing analgesia. Multimodal analgesia acts synergistically through a variety of analgesic modalities (intravenous patient-controlled analgesia, epidural analgesia, nerve block techniques, local infiltration of the incision, etc.) and analgesic drugs (opioids and nonsteroidal analgesic drugs), which in turn reduces the use of opioids and related adverse effects. ${ }^{12,29}$ Accurate analgesia requires comprehensive consideration of the patient's surgical site, size, pain intensity, and individual needs to achieve individualization of dose, route, and medication time. Because epidural analgesia is controversial in reducing postoperative adverse reactions and bringing new complications, the level of evidence recommended for its use in the relevant guidelines of ERAS is low.

\subsection{Prevention of postoperative nausea and vomiting}

Postoperative malignant vomiting is one of the main reasons for delaying gastrointestinal diet and prolonging hospital stay. Female patients and nonsmokers are prone to vomiting after surgery, and a history of motion sickness, use of inhaled anesthetics and opioids can lead to the occurrence of nausea and vomiting. ${ }^{21,30}$ At present, the prevention of such complications in clinical work mainly includes two aspects: preoperative active collection of risk factor screening and prevention, or postoperative reduction of opioid use, the use of serotonin receptor antagonists as the first-line drug for the prevention of postoperative nausea and vomiting.

\subsection{Early postoperative mobilization}

Early postoperative activities can not only reduce postoperative pneumonia, bedsore, deep venous thrombosis, and insulin resistance but also promote the recovery of intestinal tract, bladder function, body metabolism and accelerate the repair and regeneration of tissues. ${ }^{31,32}$ For this reason, targeted training should be performed before surgery to provide physiological reserve and activity confidence through the concept of pre-rehabilitation, and early activity should be achieved by postoperative evaluation including general status, motor function, nutritional status, and pain management. ${ }^{33}$

\subsection{Postoperative Catheter Care}

In the past, a drainage tube was routinely placed to drain local residual fluid after surgery to prevent infection and local irritation. Current concepts suggest that various types of abdominal drains should be used selectively rather than routinely. ${ }^{12}$ This cannot only reduce infection and other related complications but also facilitate the early postoperative movement of patients. For indwelling catheters, it can be distinguished based on urethral and nonurethral surgery, and generally nonurethral surgery advocates reducing the use of catheters or early removal. For prostatic and ileal bladder replacement, the indwelling catheter time is 1-3 weeks in order to drain urine and compression hemostasis, bladder function training. Such catheters should also be removed as much as possible based on strict care to reduce the impact on postoperative activity.

\section{Pay attention to out-of-hospital follow-up}

Out-of-hospital follow-up is a continuous treatment. The change of new nursing model attaches importance to the implementation of out-of-hospital follow-up and continuous nursing. It is mainly to understand the recovery of patients outside the hospital and whether adverse events occur. The patients are reminded to have regular reexamination in time. The patients with postoperative medication can also be informed of their drug usage, dosage, and specific course of treatment in detail. This model can reduce the postoperative readmission rate of patients and promote the rehabilitation of patients.

\section{Conclusions and prospects}

The implementation of the ERAS concept can reduce the complications and stress events caused by surgery compared with the traditional nursing model, accelerate the postoperative rehabilitation of patients, shorten the hospital stay of patients, and save medical resources. Furthermore, it can also improve the continuous care of patients outside the hospital and reduce the readmission rate of patients. The implementation of this concept has successfully revolutionized nursing technology and transformed traditional procedural nursing into whole-course nursing. However, this new concept still has the problems of insufficient specialty concreteness, small study sample, and insufficient long-term follow-up of patients. In addition, this concept also puts forward higher requirements for the overall quality of nursing staff. These advantages and challenges still suggest great potential for new ERAS concepts. 


\section{Ethical approval}

Ethical issues are not involved in this article.

\section{Conflicts of interest}

All contributing authors declare no conflicts of interest.

\section{References}

1. Steenhagen Elles. Enhanced recovery after surgery: it's time to change practice! Nutr Clin Pract 2016;31:18-29.

2. Miller TE, Thacker JK, White WD, et al. Reduced length of hospital stay in colorectal surgery after implementation of an enhanced recovery protocol. Anesth Analg. 2014;118:1052-1061.

3. Kehlet $\mathrm{H}$, Slim K. The future of fast-track surgery. $\mathrm{Br}$ J Surg. 2012;99:1025-1026.

4. Nelson G, Kiyang LN, Crumley ET, et al. Implementation of Enhanced Recovery After Surgery (ERAS) across a provincial healthcare system: the ERAS Alberta colorectal surgery experience. World J Surg. 2016;40:1092-1103.

5. Asklid D, Segelman J, Gedda C, et al. The impact of perioperative fluid therapy on short-term outcomes and 5-year survival among patients undergoing colorectal cancer surgery - a prospective cohort study within an ERAS protocol. Eur J Surg Oncol. 2017;43:1433-1439.

6. Le Roy B, Selvy M, Slim K. The concept of prehabilitation: what the surgeon needs to know? J Visceral Surg. 2016;153:109-112.

7. Tsimopoulou I, Pasquali S, Howard R, et al. Psychological prehabilitation before cancer surgery: a systematic review. Ann Surg Oncol. 2015;22:4117-4123.

8. Wilson CJ, Mitchelson AJ, Tzeng TH, et al. Caring for the surgically anxious patient: A review of the interventions and a guide to optimizing surgical outcomes. Am J Surg. 2015;212(1):151-159.

9. Zhang Z, Li H, Yan C, et al. A comparative study on the efficacy of fast-track surgery in the treatment of esophageal cancer patients combined with metabolic syndrome. Oncol Lett. 2017;14:4812-4816.

10. Apfelbaum JL, Connis RT, Nickinovich DG, et al. Practice advisory for preanesthesia evaluation: an updated report by the American Society of Anesthesiologists Task Force on preanesthesia evaluation. Anesthesiology. 2012;116:522-538.

11. AlanaziAA. Reducing anxiety in preoperative patients: a systematic review. Br J Nurs. 2014;23:387-393.

12. Cerantola $Y$, Valerio M, Persson $B$, et al. Guidelines for perioperative care after radical cystectomy for bladder cancer: enchanced recovery after surgery(ERAS) society recommendation. Clin Nutr. 2013;32:879-887.
13. Wong J, Lam DP, Abrishami A, et al. Short-term preoperative smoking cessation and postoperative complications: a systematic review and meta-analysis. Can J Anaesth. 2012;59:268-279.

14. Pierre S, Rivera C, Le Maître B, et al. Guidelines on smoking management during the perioperative period. Anaesth Crit Care Pain Med. 2017;36:195-200.

15. Czoski-Murray C, Lloyd JM, McCabe C, et al. What is the value of routinely testing full blood count, electrolytes and urea, and pulmonary function tests before elective surgery in patients with no apparent clinical indication and in subgroups of patients with common comorbidities: a systematic review of the clinical and cost-effective literature. Health Technol Assess. 2012;16:1-159.

16. Dexter F, Wachtel RE. Strategies for net cost reductions with the expanded role and expertise of anesthesiologists in the perioperative. Surg Home Anesth Analg. 2014;118:1062-1071.

17. Mcclave SA, Kozar R, Martindale RG, et al. Summary points and consensus recommendations from the north american surgical nutrition summit. J Parenter Enteral Nutr. 2013;37(5 Suppl): 99S-105S.

18. Gillis C, Fenton T R, Sajobi TT, et al. Trimodal prehabilitation for colorectal surgery attenuates post-surgical losses in lean body mass: a pooled analysis of randomized controlled trials. Clin Nutr, 2019;38:1053-1029.

19. $\mathrm{Xu} \mathrm{D}, \mathrm{Zhu} X, \mathrm{Xu} Y$, et al. Shortened preoperative fasting for prevention of complications associated with laparoscopic cholecystectomy: a meta-analysis. J Int Med Res. 2017;45:22-37.

20. Feng C, Sidhwa F, Anandalwar S, et al. Contemporary practice among pediatric surgeons in the use of bowel preparation for elective colorectal surgery: a survey of the American Pediatric Surgical Association. Pediatr Surg. 2015;50:1636-1640.

21. Gustafsson UO, Scott MJ, Hubner M, et al. Guidelines for perioperative care in elective colorectal surgery: enhanced recovery after surgery (ERAS) society recommendations: 2018. World J Surg. 2019;43:659-695.

22. Lee JS, Song Y, Kim JY, et al. Effects of preoperative oral carbohydrates on quality of recovery in laparoscopic cholecystectomy: a randomized, 
double blind, placebo-controlled trial. World J Surg. 2018;42:3150-3157.

23. Austin PN. Forced-air warmers and surgical site infections in patients undergoing knee or hip arthroplasty. Annu Rev Nurs Res. 2017;35:179-199.

24. Steelman VM, Chae S, Duff J, et al. Warming of irrigation fluids for prevention of perioperative hypothermia during arthroscopy: a systematic review and meta-analysis. Arthroscopy. 2018;34: 930-942.

25. Elhassan A, Elhassan I, Elhassan A, et al. Essential elements for enhanced recovery after intra-abdominal surgery. Curr Pain Headache Rep. 2019;23:35.

26. Zhu AC, Agarwala A, Bao X. Perioperative fluid management in the enhanced recovery after surgery (ERAS) pathway. Clin Colon Rectal Surg. 2019;32:114-120.

27. Feng S, Yang S, Xiao W, et al. Effects of perioperative goal-directed fluid therapy combined with the application of alpha-1 adrenergic agonists on postoperative outcomes: a systematic review and metaanalysis. BMC Anesthesiol. 2018;18:113-126.

28. Villafranca A, Thomson IA, Grocott HP, et al. The impact of bispectral index versus end-tidal anesthetic concentration-guided anesthesia on time to tracheal extubation in fast-track cardiac surgery. Anesth Analg. 2013;116:541-548.

29. Xu W, Daneshmand S, Bazargani ST, et al. Postoperative pain management after radical cystectomy comparing traditional versus enhanced recovery protocol pathway. J Urol. 2015;194: 1209-1213.

30. Apfel CC, Heidrich FM, Jukar-Rao S, et al. Evidence-based analysis of risk factors for postoperative nausea and vomiting. $\mathrm{Br} J$ Anaesth. 2012;109:742-753.

31. Van der Leeden M, Huijsmans R, Geleijn E, et al. Early enforced mobilisation following surgery for gastrointestinal cancer: feasibility and outcomes. Physiotherapy. 2016;102:103-110.

32. Hanada M, Kanetaka K, Hidaka S, et al. Effect of early mobilization on postoperative pulmonary complications in patients undergoing video-assisted thoracoscopic surgery on the esophagus. Esophagus. 2018;15:69-74.

33. Hodgson CL, Capell E, Tipping CJ. Early mobilization of patients in intensive care: organization, communication and safety factors that influence translation into clinical practice. Crit Care. 2018;22:77. 